\title{
Rotational and domain wall motion aftereffect in a patterned array of small particles
}

\author{
M. Pardavi-Horvath ${ }^{\mathrm{a})}$ \\ Department of ECE, The George Washington University, Washington, District of Columbia 20052
}

G. Vertesy, B. Keszei, Z. Vertesy

Research Institute for Technical Physics and Materials Science, H-1525, Budapest, POB 49, Hungary

\begin{abstract}
Aftereffect for magnetization processes by rotation and by domain wall motion was investigated on the same, single domain, two-state system of a square 2D (two-dimensional) array of garnet particles. Aftereffect measurements were performed magnetooptically. The particles are thermally stable, the particle energy is $10^{-6} \mathrm{erg}$ compared to the thermal energy of $10^{-12} \mathrm{erg}$. No aftereffect of rotation switching of the system of "up" and "down" magnetized particles could be observed at room temperature. At increased temperatures thermally activated switching, very weakly depending on magnetic field, is observed. Each individual particle can be demagnetized into a metastable stripe domain structure. The barrier for DW (domain wall) motion is much lower than the barrier for rotation, and a significant aftereffect was measured on the same particles, demagnetized into a domain structure. The observed time dependence for DW aftereffect is exponential, $M(t) / M s=d^{*} \exp (-e t)$, where $d=0.04$, and $e=1 / \tau$ follows the increase of the magnetization with field, deldH=0.021/s/Oe. (C) 2000 American Institute of Physics.
\end{abstract}

[S0021-8979(00)61308-7]

\section{INTRODUCTION}

Patterned magnetic nanostructures are the best candidates for future extreme high density magnetic recording media. Most of the fundamental problems, related to the application of the patterned structures, can be conveniently studied on a two-state model system of a square array of magnetically small, single crystal, strongly uniaxial, square garnet particles. ${ }^{1-3}$ One of the major concerns about patterned media is the thermal and temporal instability of the information, due to the low anisotropy energy $E_{K}=K V$ of the small volume $(V)$ particles with respect to the fluctuations of thermal energy. ${ }^{4}$ However, the stability of different magnetic states of the same system is different. ${ }^{5}$ The barrier with respect to the mechanism of the change of the magnetization, i.e., rotation vs domain wall (DW) motion is different too. The sensitivity of the magnetic aftereffect to the type of the dominant reversal mechanism was shown on Co-based nanomultilayers. ${ }^{6}$ In continuous, or bulk magnetic materials, even in the case when the dominant mechanism is rotation, DW motion processes are always present around the coercivity. This gives rise to the characteristic maximum on the $S$ $=d M / d \ln t$ vs $H$ curves. It is assumed, that for materials where DW processes are excluded, this curve is more flat. The same behavior is expected for a patterned recording medium of high anisotropy particles.

In the present work the particles on the $2 \mathrm{D}$ array are thermally very stable, as the anisotropy field $H_{K}=2 \mathrm{kG}$, $K_{u}=1.3 \times 10^{4} \mathrm{erg} / \mathrm{cm}^{3}, V=7.5 \times 10^{-9} \mathrm{~cm}^{3}$, thus $E_{K} \approx 10^{-4}$ erg in $H=0$. The strong uniaxial anisotropy causes the particles to be in the single domain state. It is not expected to observe any aftereffect of switching by rotation. However,

${ }^{a)}$ Electronic mail: pardavi@seas.gwu.edu upon increasing the temperature the anisotropy barrier is lowered, the temperature fluctuations increase, and it is expected that the aftereffect of the rotational switching of the magnetization can be observed.

The same system of 2D array of particles can be used to study the DW motion aftereffect. The barrier for DW motion is much lower than the barrier for rotation. It is expected that the aftereffect, related to magnetization change by DW motion, can easily be observed for the same system in a metastable stripe domain demagnetized state.

\section{EXPERIMENT}

The investigated system is a square $2 \mathrm{D}$ array of uniform, uniaxial, single crystalline particles. The $1 \mathrm{~cm} \times 1 \mathrm{~cm} 2 \mathrm{D}$ array of $50 \mu \mathrm{m}$ square particles is etched from a $3 \mu \mathrm{m}$ thick, epitaxial garnet layer. The particles are separated by $16 \mu \mathrm{m}$ wide nonmagnetic grooves. The magnetization $4 \pi M_{s}=160$ $\mathrm{G}$, is low compared to the anisotropy field $H_{u}=2 \mathrm{kOe}$. As a result, there are only two stable magnetic states, either up or down along the easy axis, normal to the film plane. Figure 1(a) illustrates four particles of the system in $H=0$ in the $a c$ demagnetized state. The coercivity of the major loop $H_{C}$

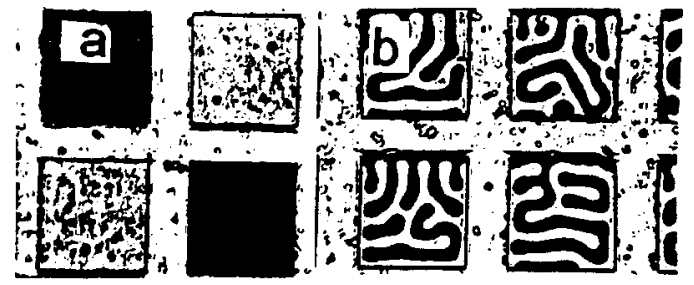

FIG. 1. (a) ac demagnetized 2D array of single domain particles in $H=0$; (b) stripe domain demagnetized metastable state of the same system. Optical micrograph in polarized light (Faraday effect). 


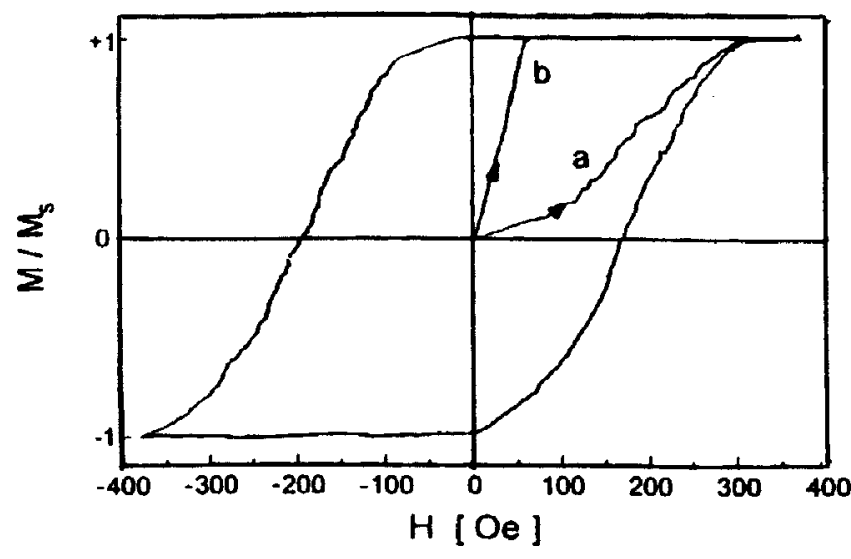

FIG. 2. Room temperature major hysteresis loop and initial magnetization curves for: (a) demagnetized sample with single domain particles (checkerboard pattern), (b) stripe domain demagnetized particles.

$=280$ Oe. Each particle has a rectangular hysteresis loop, and the switching of the whole system proceeds by a sequence of incoherent rotation of individual particles. ${ }^{1,3}$

However, the ground state of the epitaxial garnet layer before structuring is a stripe domain structure of $10 \mu \mathrm{m}$ periodicity. By saturating the sample in a very large in-plane magnetic field, the particles can be individually demagnetized into a stripe domain (SD) structure, as shown in Fig. 1(b). This domain structure in the structured material is unstable, and upon application of a moderate field, normal to the film plane, it disappears, and the system returns to the "checkerboard" pattern, stable along either the major or any minor hysteresis loop (Fig. 2).

Measurements of the time, field, and temperature dependence of the aftereffect have been performed both on the checkerboard pattern, switching by rotation, and on the SD demagnetized sample, where the magnetization process is by domain wall (DW) motion. All the measurements were done magneto optically, in the Faraday geometry on single particles or on arbitrary groups of particles.

The rotational aftereffect was measured on previously saturated single domain particles. The number of particles (about 80) was chosen such a way, that the switching of the individual pixels could be detected, i.e., the intensity change, caused by switching of one particle, is larger than the noise of the measured signal.

Measurements of the DW motion aftereffect were done on stripe domains by applying field steps up to $27 \mathrm{Oe}$; and at different temperatures, applying the same field step of 20 Oe. It is found that the $\tau_{s}$ time to reach saturation of the aftereffect, as a function the field strength and temperature, is a reliable characteristics of the process.

\section{RESULTS AND DISCUSSION}

Due to the high particle energy, no rotational aftereffect has been observed at room temperature, as expected. The switching fields of individual particles have a rather broad Gaussian distribution, as discussed in Ref. 3. The lowest switching field is about $100 \mathrm{Oe}$, the highest is about $500 \mathrm{Oe}$. If to assume that: The barrier height $H_{b}$ is lowered the most at the lowest switching field; the incoherent switching vol-

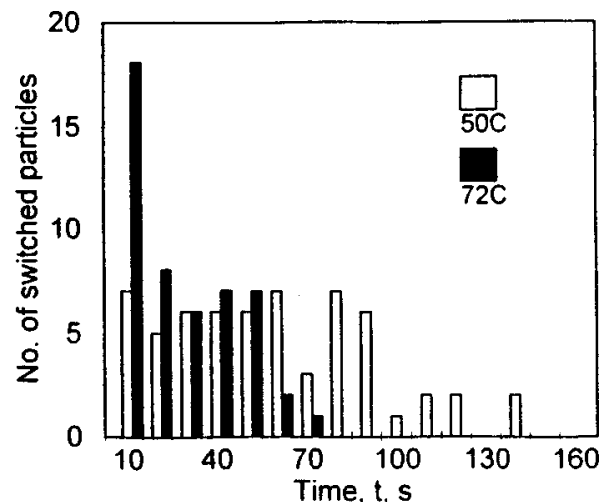

FIG. 3. Rotational aftereffect. Number of single domain particles, switched in $10 \mathrm{~s}$ intervals in $H=H_{c} .\left(H_{c}=164 \mathrm{Oe}\right.$ at $T=50^{\circ} \mathrm{C} ; H_{c}=128.5 \mathrm{Oe}$ at $72{ }^{\circ} \mathrm{C}$.)

ume is a $0.8 \mu \mathrm{m}$ "rim" of the particle (corresponding to the activation volume $V_{a}$ ) ; the applied field is $H_{c}$; and the thermal fluctuations correspond to $40 k_{B} T=E_{T} \approx 10^{-12} \mathrm{erg}$ and $E_{K}=K_{u} V_{a}\left(1-H / H_{b}\right)^{2}=10^{-6} \mathrm{erg}-\mathrm{it}$ is still too large for any observable aftereffect.

Upon increasing the temperature, the coercivity is decreasing $\left(T=50^{\circ} \mathrm{C}: H_{c}=164\right.$ Oe; $T=72{ }^{\circ} \mathrm{C}: H_{c}=128.5$ $\mathrm{Oe})$; thermal fluctuations increase, and it is possible to observe the rotational aftereffect. The time evolution of the number of particles, switched during $10 \mathrm{~s}$ intervals, in $H$ $=H_{c}$, is plotted in Fig. 3. This plot reflects the distribution of the barrier heights of the particles. At $50{ }^{\circ} \mathrm{C}$ the time distribution of particle's switching is rather even, the "best" particle, switching the last (140 s) has the highest barrier. At $72{ }^{\circ} \mathrm{C}$ the switching proceeds faster, the process ends after 70 s. At room temperature no particle was switched during 5 min.

The time dependence of the normalized sum of the number of particles switched in $H$ is shown in Fig. 4. As it was seen in Fig. 3, the process of switching is faster at elevated temperatures. The two other sets of measurements at $50^{\circ} \mathrm{C}$, taken at different magnetic fields, does not differ significantly. For the present system the $M=0$ demagnetized state at $H_{c}=280 \mathrm{Oe}$ is still consists of up and down magnetized

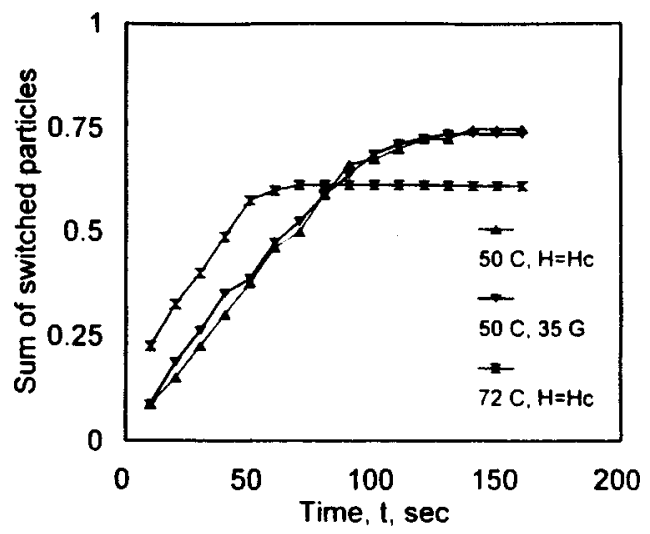

FIG. 4. Temperature and magnetic field dependence of the rotational aftereffect. Total number at particles switched at a given time. 
single domain particles, and the aftereffect is governed by stability against rotation at any field.

However, there is a metastable demagnetized state of lower energy, when each individual particle is demagnetized into a stripe domain pattern. Figure 2 illustrates the initial magnetization curves for both cases. In the stripe domain state the DW motion coercivity is 0.8 Oe. This barrier for DW motion is much lower than the barrier for rotation, and a strong aftereffect is expected and observed. The time dependence of the DW aftereffect is exponential, $M(t) / M_{s}$ $=d^{*} \exp (-e t)$, where $d \cong 0.04$. The decay coefficient $e(H)$ $=1 / \tau \sim M(H) / M_{s}$, follows the increase of the magnetization with field with $d e / d H=0.021 / \mathrm{s} / \mathrm{Oe}$. The temperature dependence of the time $\tau_{s}$ when the magnetization reaches its equilibrium value, $d M / d t=0$ in $H=20 \mathrm{Oe}$, is about $0.15 \mathrm{~s} / \mathrm{K}$ compared to the $3 \mathrm{~s} / \mathrm{K}$ for magnetization change by rotation.

The aftereffect depends on the shape and size of particles. It is observed that $\tau_{s}$ of DW motion is longer for smaller particles with higher aspect ratio $A$, i.e., for $A=$ thickness $/$ width $=3 / 40=0.075 \tau_{s}=2 \mathrm{~s} / \mathrm{G}$, and $\tau_{s}=0.3 \mathrm{~s} / \mathrm{G}$ for $A=3 / 60=0.05$, as the internal field is more homogeneous in the "more flat" particles.

\section{CONCLUSIONS}

Magnetic aftereffect for magnetization processes by rotation and by domain wall motion was investigated on the same two-state system of garnet particles on a 2D array. The particles are thermally stable, at room temperature and no aftereffect of rotation could be observed. The same behavior is expected for a patterned recording medium of high anisotropy particles. At elevated temperatures, where the anisot- ropy is reduced and thermal fluctuations increase, it is shown that the aftereffect of the 2D array of single domain particles does not depend on the applied field due to the lack of DW motion processes.

There is a metastable demagnetized state of lower energy, when each individual particle is demagnetized into a stripe domain pattern. The barrier for DW motion is much lower than the barrier for rotation, and a significant DW aftereffect was measured on the same particles. The observed time dependence is strictly exponential, and the decay coefficient is proportional to the magnetization. The relaxation process depends on the shape and size of the particles.

It is shown that magnetic relaxation is qualitatively different for magnetization by domain wall motion and rotation. Whenever the magnetization is changing by a combination of the rotation and DW motion, the characteristic bellshaped magnetic field dependence of the viscosity coefficient, due to the combined effect, is observed.

\section{ACKNOWLEDGMENTS}

Support by NSF Grant Nos. DMR-9806050 (MPH) and OTKA T-026153 is greatly acknowledged.

\footnotetext{
${ }^{1}$ M. Pardavi-Horvath and G. Vertesy, IEEE Trans. Magn. 30, 124 (1994).

${ }^{2}$ M. Pardavi-Horvath, IEEE Trans. Magn. 32, 4458 (1996).

${ }^{3}$ M. Pardavi-Horvath, G. Vertesy, B. Keszei, Z. Vertesy, and R. D. McMichael, IEEE Trans. Magn. 35, 3871 (1999).

${ }^{4}$ S. M. Stinnett, W. D. Doyle, P. J. Flanders, and C. Dawson, IEEE Trans. Magn. 34, 1828 (1998).

${ }^{5}$ M. Pardavi-Horvath, J. Oti, G. Vertesy, E. Kisdi-Koszo, L. H. Bennett, and L. J. Swartzendruber, J. Magn. Magn. Mater. 104-107, 313 (1992).

${ }^{6}$ S.-C. Shin and S.-B. Choe, IEEE Trans. Magn. 35, 3853 (1999).

${ }^{7}$ M. Pardavi-Horvath, J. Magn. Magn. Mater. 198-199, 219 (1999).
} 